\title{
Los minicasos como un método docente adyuvante a las clases magistrales: la opinión de los estudiantes sobre una experiencia piloto en farmacología
}

\author{
Minicases as an adjunct to lectures in the teaching of pharmacology. \\ The students' point of view in a pilot experience.
}

Magí Farréi,2 y Josep-Eladi Baños ${ }^{3 *}$

${ }^{1}$ Departamento de Farmacología, de Terapéutica y de Toxicología. Universitat Autònoma de Barcelona. ${ }^{2}$ Departamento de Farmacología. Institut Municipal d'Investigació Mèdica. Barcelona. ${ }^{3}$ Departamento de Ciencias Experimentales y de la Salud. Universitat Pompeu Fabra. Barcelona.

Introducción: En el presente artículo describimos un estudio piloto utilizando los minicasos, conjuntamente con las clases magistrales, en la docencia de la farmacología.

Material y Métodos:. Se utilizaron 9 minicasos. Consistían en un texto de 5 líneas con dos preguntas, relacionadas con el tema de la clase magistral. Se entregaban al inicio de ésta y se concedía a los estudiantes 5 minutos al finalizarla para responderlas. La participación era voluntaria. Los textos se corregían y se podía obtener hasta 1 punto adicional a la nota del examen. Se evaluó la opinión de los estudiantes mediante una encuesta diseñada al efecto. Resultados: De los 69 estudiantes matriculados 43 respondieron. Entregaron una media de 7,6 minicasos y puntuaron 7,5 su utilidad para entender mejor la disciplina, 5,9 el grado de dificultad y 7,3 su interés. Sobre las razones en participar, puntuaron 8,5 la posibilidad de obtener el extrabonus, 7,5 el conocer mejor los temas y 6,8 el desafío de resolver el problema. El $67 \%$ señaló que habían aumentado su asistencia a las clases magistrales y el $95 \%$ que los resolverían sin extrabonus. Todos participarían en experiencias futuras de este tipo.
Correspondencia:

Dr. Josep-E. Baños

Departamento de Ciencias Experimentales y de la Salud.

Universitat Pompeu Fabra

Dr. Aiguader 80. 08003-Barcelona.

Teléfono 935422950

josepeladi.banos@upf.edu
Conclusiones: Los minicasos pueden ser un método eficiente para mejorar la asistencia de los estudiantes a las clases magistrales y mejorar la comprensión de éstas.

Palabras clave: Clases magistrales, farmacología, enseñanza de la medicina, minicasos.

Introduction: We report here a preliminary study using mini-cases or very short problems in conjunction with traditional lectures for teaching medical students. Material and Methods: We used nine mini-cases in our lectures on pharmacology. Mini-cases consist of a five-line text with two questions on the subject of the lecture. They were delivered just before the lecture, and students were given five minutes to answer the questions at the end. Teachers evaluated their correctness and were able to award up to 1 extra point to the overall mark in the regular exam. At the end of term the experience was evaluated by asking students to answer ten questions anonymously.

Results: Forty-three (out of 69) answered the questionnaire. They handed in a mean of 7.6 mini-cases and gave a mean score of 7.5 for their usefulness in understanding the subject better, $\mathbf{5 . 9}$ for the degree of difficulty and $\mathbf{7 . 3}$ for their interest. When asked about their reasons for taking part, they scored 8.5 for the possibility of obtaining an extra mark, 7.5 for the idea that they would understand the subjects better and 6.8 for the challenge of solving the problem. Around $67 \%$ reported that mini-cases increased attendance at lectures and $95 \%$ said they would participate even if 
extra marks were not available. All participants reported that they would participate in similar experiences in the future.

Conclusion: We conclude that mini-cases may be a cost-effective method for improving students' attendance at lectures, as well as their understanding.

Key words: lectures, pharmacology, teaching of medicine, mini-cases.

\section{INTRODUCCIÓN}

Durante muchos años las clases magistrales han constituido el principal recurso docente de las facultades de medicina con una dedicación horaria muy superior a otro tipo de actividades si se exceptúa, en contadas ocasiones, las actividades prácticas. A pesar de que su valor pedagógico como método docente predominante ha sido motivo de crítica constante, es poco creíble que se reduzcan de forma substancial en el futuro inmediato, a pesar de que diversos autores han propuesto otros métodos que estimulen más la participación activa de los estudiantes ${ }^{1,2}$.

Entre los métodos docentes propuestos para mejorar el aprendizaje de los estudiantes, figuran aquellos que les permiten contextualizar el conocimiento mediante su exposición a situaciones reales o imaginarias. El caso clínico constituye en medicina el paradigma de este método, pero precisa que los estudiantes dispongan de un bagaje de conocimientos en el ámbito médico que se encuentra ausente en los primeros años de la licenciatura. El aprendizaje basado en problemas es asimismo un método que ha gozado de gran predicamento, pero su empleo se encuentra limitado por sus propias características conceptuales y la necesidad de desarrollarlo en grupos pequeños ${ }^{3}$. A pesar de que tales métodos tienen su indudable utilidad, hay pocas dudas de que las clases magistrales persistirán durante largo tiempo.

En numerosas facultades, la docencia basada en clases magistrales se enfrenta al absentismo de los estudiantes $^{4}$, que prefieren dedicar su tiempo a otras actividades y evitan permanecer durante varias horas recibiendo información más o menos relevante para lo que creen que debe ser su formación. Esta ausencia puede deberse, al menos en parte, a la pasividad que imponen las clases magis- trales. Existen estudios que muestran que la asistencia a clase mejora, sin embargo, el rendimiento académico $^{5}$, por lo que deberían explorarse aquellos métodos que permitan aumentarla.

El presente estudio describe una experiencia piloto realizada con el empleo de los minicasos como adyuvante de las clases magistrales. Su objetivo fue establecer si mejoraba la asistencia a clase y evaluar la aceptación por parte de los estudiantes.

\section{MÉTODOS}

La experiencia se realizó en el marco de la asignatura de Farmacología correspondiente a tercer curso de la licenciatura en Medicina. El programa contiene 52 temas teóricos y la docencia está repartida en dos cuatrimestres. Se llevó a cabo durante el primer cuatrimestre del curso 2004-2005 en el que había 69 alumnos matriculados en la asignatura de los cuales 57 en primera convocatoria y 12 en las sucesivas. Estos últimos no suelen asistir a clase por coincidencia horaria con otras asignaturas de cuarto curso.

Los minicasos son textos de unas cinco líneas de extensión que describen una situación imaginaria que está relacionada con un tema de farmacología e incluyen con dos preguntas relacionadas con el texto. En esta experiencia se utilizaron nueve minicasos escritos por los autores.

El procedimiento fue siempre el mismo. Antes del inicio de la clase magistral se entregaba a los estudiantes presentes una hoja con el texto del minicaso y las preguntas. Se leía conjuntamente y se procedía a iniciar la clase que trataba de conceptos que permitían entender el minicaso y contestar las preguntas planteadas. Todos estaban relacionados con temas de farmacología del sistema nervioso periférico y de mediadores de la inflamación. La Figura 1 describe uno de ellos. Al finalizar la clase, el profesor concedía cinco minutos a los estudiantes para proceder a la respuesta escrita de las preguntas. Pasado este tiempo, se recogían los textos y se daban las respuestas correctas, con un comentario posterior si era oportuno. Los estudiantes desconocían el calendario de presentación de los minicasos.

La participación en la experiencia fue voluntaria y los estudiantes podían obtener hasta 1 punto adicional (extrabonus) a su nota en el examen regular del cuatrimestre. La asignación del extrabonus se realizaba tras la corrección de las respuestas por los profesores. Al final del cuatrimestre, se pidió a los estudiantes que habían participado en la experiencia que respondieran voluntaria y anónimamente 


\section{Tabla 1: Encuesta de evaluación de los minicasos}

- ¿Cuántos minicasos has entregado durante el cuatrimestre?

- ¿Cuál ha sido la utilidad de los minicasos para entender mejor la asignatura? ( $0=$ nada, 10=mucho).

- ¿Cuál ha sido el nivel de dificultad de los problemas? ( $0=$ nada, 10=mucho).

- ¿Cuál ha sido el interés de los problemas para entender la farmacología? ( $0=$ nada, 10=mucho).

- Puntúa de 0 (nada) a 10 (mucho) el motivo por el que has entregado los minicasos?: 1.- La posibilidad de obtener

un extrabonus. 2.- El reto de solucionar un problema. 3.- La creencia de que me ayuda a aprender Farmacología

- La realización de los minicasos ha aumentado tu asistencia a clase (SI/NO).

- ¿Realizarías la actividad si no se concediera el extrabonus? (SI/NO).

- ¿Crees que la evaluación debería incluir minicasos? (SI/NO).

- ¿Alguna sugerencia para mejorar la actividad?

una encuesta de 10 preguntas sobre diversos aspectos relacionados con la experiencia (Tabla 1). Las puntuaciones otorgadas oscilaban entre 0 y 10 .

\section{RESULTADOS}

Respondieron la encuesta 43 de los 69 alumnos matriculados $(62 \%)$. Si se consideran los matricula-

\section{Figura 1: El minicaso Barça - Madrid}

Pedro es socio del Barça desde que nació. Es "culé" hasta la médula. Durante la primera parte del partido Barça-Madrid tiene los nervios a flor de piel y nota que el corazón le late muy rápidamente. Al final de la primera parte, le comenta a su compañero de la grada que siente palpitaciones. Este le dice: "iA ver si tendrás un infarto!, tómate una de estas pastillas y verás como se te pasa". Pedro se las toma. Este es el registro de su frecuencia cardiaca durante el partido:

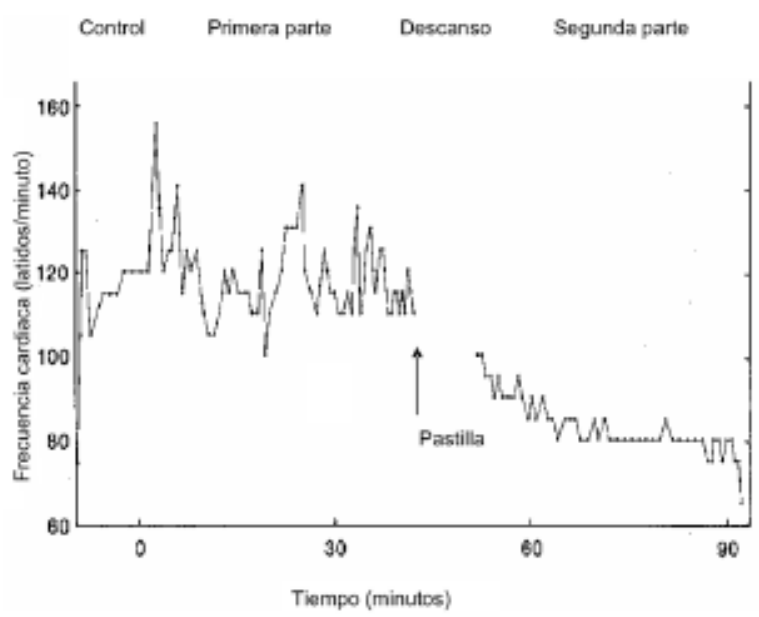

- ¿Qué tipo de fármaco crees que contiene la pastilla?

- Si Pedro fuera asmático, ¿qué podría haberle pasado? dos en primera convocatoria, el porcentaje de respuesta ascendió al 83\%. Los participantes habían respondido un promedio de 7,6 minicasos durante el cuatrimestre y 22 de ellos (32\%) los habían completado todos. El número de textos recogidos osciló entre 38 y 43 durante la experiencia. Un $67 \%$ respondieron que su asistencia a clase había aumentado desde que se empezaron a utilizar los minicasos.

$\mathrm{Al}$ ser preguntados sobre la utilidad de los minicasos para entender mejor la asignatura, los estudiantes otorgaron una puntuación (media \pm desviación estándar) de 7,5 $\pm 1,5$ mientras que dieron un valor de $7,3 \pm 1,6$ en su interés y de $5,9 \pm 1,6$ en la dificultad para resolverlos. Las puntuaciones otorgadas individualmente a los casos oscilaron entre $6,8 \pm 1,6$ y $7,6 \pm 1,6$.

Preguntados sobre las razones para participar en la experiencia, la posibilidad de obtener un extrabonus fue puntuada con $8,5 \pm 2,4$. Sin embargo, un $95 \%$ señaló que participaría en la experiencia aunque no se proporcionara tal puntuación adicional. Asimismo, concedieron una puntuación de 7,5 $\pm 1,6$ al interés de los minicasos en comprender mejor la disciplina y $6,8 \pm 2.2$ al reto de resolver un problema. Todos los estudiantes manifestaron que volverían a participar en experiencias similares en el futuro.

\section{DISCUSIÓN}

A pesar de los numerosos avances en el ámbito de las tecnologías de la información y del gran trabajo pedagógico desarrollado en las últimas décadas en las técnicas de aprendizaje activo, es indudable que la clase magistral sigue ocupando buena parte de las actividades docentes de numerosos profesores de las facultades de Medicina españolas. Existen diversas explicaciones para esta situación, unas relacionadas con los profesores, otras con los alumnos o con las propias instituciones. Entre las 
primeras se cuentan la dificultad de implementar nuevos métodos en un entorno llamémosle "hostil" o la resistencia a los cambios de rol docente. En los alumnos, el esfuerzo requerido por los métodos más activos es un motivo notable de resistencia, así como la costumbre inaceptablemente extendida de realizar los estudios universitarios como los antiguos "alumnos libres", debido al elevado absentismo de todas aquellas actividades donde no se realice un control de asistencia ${ }^{4-6}$. Las instituciones tampoco lo ponen fácil con planes de actividad docente claramente burocráticos, aulas inadaptadas para las necesidades, pocos recursos para el cambio y, con excesiva frecuencia, una desincentivación de la actividad docente frente a la investigadora ${ }^{2}$.

En la situación expuesta en el párrafo anterior no es pues difícil considerar la gran dificultad de cambiar de paradigma docente de la noche a la mañana, especialmente en centros de larga tradición. Por ello, haciendo de la necesidad virtud, deberían implementarse métodos que permitan que la clase magistral adopte algunos elementos que la hagan más atractiva a los estudiantes. No hay duda que una buena clase magistral genera una elevada motivación en los alumnos, pero el problema es que su abuso y la escasez de clases auténticamente "magistrales" acaban por aburrir, especialmente cuando las pruebas de evaluación están destinadas a establecer los conocimientos factuales de los alumnos, que pueden obtenerse mediante otros métodos ${ }^{5}$.

La experiencia que se describe en el presente artículo quiere combinar la reflexión y la pregunta con la exposición tradicional a fin de permitir contextualizar el conocimiento y trasmitir a los estudiantes el interés de los conceptos que se exponen. Los resultados obtenidos son positivos si consideramos que dos de cada tres estudiantes manifestó que los minicasos habían aumentado su asistencia (voluntaria) a clase, sin descartar que, quizá, parte del resto ya asistía regularmente. Los estudiantes fueron sinceros al manifestar que la razón más importante para participar en la experiencia fue la obtención del extrabonus, pero no debe despreciarse la puntuación otorgada al hecho de que los minicasos les ayudaban a comprender mejor la asignatura, lo que era coherente con la puntuación que daban al interés propio de la experiencia. En cualquier caso, una gran mayoría reveló que participaría aunque no existiera el premio de la puntuación adicional. Estos datos pueden considerarse como altamente satisfactorios, sobre todo si se considera la relación coste-beneficio del empleo de los minica- sos, en realidad un poco de imaginación, algunas fotocopias y el tiempo de la corrección.

La mejoría de la asistencia a clase no es la única consecuencia beneficiosa de los minicasos. Como han señalado Lubawy y Brandt ${ }^{7}$, este método permite que los estudiantes aprecien que la farmacología ayuda a entender situaciones reales y no es tan sólo una disciplina de conocimiento teórico. Puede constituir, además, el principio para enseñar a los estudiantes cómo conjugar conceptos y resolución de escenarios en una fase inicial de su formación ${ }^{8}$.

En conclusión, la experiencia mostró que los minicasos pueden ser un método eficiente para mejorar la asistencia de los estudiantes a las clases magistrales y mejorar, en su opinión, la comprensión de éstas. Los resultados de la encuesta muestran que tiene una aceptación suficiente por los estudiantes. Todos los resultados deben considerarse con precaución, dada el carácter preliminar del estudio, pero constituyen un primer paso para una evaluación posterior más amplia y extensa. Queda asimismo por dilucidar si los minicasos también mejoran el rendimiento académico.

\section{BIBLIOGRAFÍA}

1. Rozman C. La educación médica en el umbral del siglo XXI. Med Clin (Barc) 1997; 108:582-586.

2. Baños JE. El aprendizaje basado en problemas en los planes de estudio tradicionales: ¿una alternativa posible? Educ Med 2001; 4:4-12.

3. Rodríguez J, Higuera FJ, de Anda E. Aprendizaje basado en problemas. México: Panamericana, 2002.

4. Ortega-Serrano J, Martínez-León J, Aliaga-Estellés J. Influencia de la asistencia a clases teóricas y otros factores en el rendimiento académico de alumnos pregraduados en Cirugía. Rev Clin Esp 1990; 187:241-246.

5. Pérez J. Graell S. Asistencia a clase y rendimiento académico en estudiantes de medicina: la experiencia de la Universidad Autónoma de Barcelona. Educ Med 2004; 7:8589.

6. Sitges-Serra A, Mingella JL, Carulla X et al. Relación entre hábitos de estudio, nota y valoración del profesorado: encuesta a 38 estudiantes de Patología Quirúrgica I. Acta Chir Catal 1998; 9:253.

7. Lubawy WC, Brandt BF. A variable structure, less resource intensive modification of problem-based learning for pharmacology instruction to health science students. NaunynSchmiedeberg's Arch Pharmacol 2002; 366:48-57.

8. Lubawy WC, Brandt BF. Microsituations as an active-learning tool to teach endocrine pharmacology and problem-solving skills. Am J Pharm Educ 1998; 62:333-341. 